\title{
Adjuvantes no Tratamento da Hiperglicemia do Diabetes Melito Tipo 1
}

\begin{abstract}
RESUMO
Desde o Diabetes Control and Complications Trial (DCCT), a terapia insulínica intensiva tem sido direcionada para alcançar valores de glicemia e hemoglobina glicada ( $\mathrm{HbA1c}$ ) tão próximos do normal quanto a segurança permita. Entretanto, a hiperglicemia (especialmente a hiperglicemia pós-prandial) e a hipoglicemia continuam a ser um problema no manejo do diabetes tipo 1.0 objetivo de associar outras drogas à terapia insulínica é diminuir a glicemia pós-prandial. A terapia adjunta pode ser dividida em três grupos, conforme seu mecanismo de ação: 1 . Aumento da ação da insulina (metformina e tiazolidinedionas); 2 . Alteração da liberação de nutrientes no trato gastrintestinal (acarbose e amilina); 3. Outros modos de ação [pirenzepina, fator de crescimento insulina-símile (IGF-1) e peptídeo semelhante ao glucagon 1 (GLP-1). Muitos desses agentes mostraram, em estudos de curto prazo, diminuição de $0,5 \%$ a $1 \%$ na $\mathrm{HbA} 1 \mathrm{c}$, diminuir a hiperglicemia pós-prandial e as doses diárias de insulina. (Arq Bras Endocrinol Metab 2008;52/2:279-287)
\end{abstract}

Descritores: Diabetes tipo 1; Hiperglicemia; Terapêutica; Adjuvantes

\begin{abstract}
Adjunctive Therapies to Glycaemic Control of Type 1 Diabetes Mellitus. Since Diabetes Control and Complications Trial (DCCT), intensive therapy has been directed at achieving glucose and glycosylated hemoglobin (HbA1c) values as close to normal as possible regarding safety issues. However, hyperglycemia (especially postprandial hyperglycemia) and hypoglicemia continue to be problematic in the management of type 1 diabetes. The objective of associating other drugs to insulin therapy is to achieve better metabolic control lowering postprandial blood glucose levels. Adjunctive therapies can be divided in four categories based on their mechanism of action: enhancement of insulin action (e.g. the biguanides and thiazolidinediones), alteration of gastrointestinal nutrient delivery (e.g. acarbose and amylin) and other targets of action (e.g. pirenzepine, insulin-like growth factor I and glucagon-like peptide-1). Many of these agents have been found to be effective in shortterm studies with decreases in $\mathrm{HbA} 1 \mathrm{c}$ of $0.5-1 \%$, lowering postprandial blood glucose levels and decreasing daily insulin doses. (Arq Bras Endocrinol Metab 2008;52/2:279-287)
\end{abstract}

Keywords: Type 1 diabetes; Hyperglycemia; Treatment; Adjunctive agents

\section{INTRODUÇÃO}

$\mathrm{D}$ ESDE A DESCOBERTA DA INSULINA por Banting e Best em 1921, muitas mudanças ocorreram em sua produção e no modo de utilização, revolucionando o tratamento insulínico dos indivíduos com diabetes melito tipo 1 (DMl). Os avanços tecnológicos aliados a grandes estudos, como o Diabetes revisõo

Mônica de A. Lima Gabbay

Disciplina de Endocrinologia do Departamento de Medicina da Escola Paulista de Medicina da Universidade Federal de São Paulo (EPM/Unifesp), SP, Brasil.
Recebido em 30/1 1/2007

Aceito em 10/12/2007 
Control and Complications Trial (DCCT) (1) confirmam a importância da terapia insulínica intensiva, seja por meio de múltiplas doses de insulina ou por intermédio da bomba de infusão de insulina subcutânea para se atingir a euglicemia. No entanto, o ganho de peso e a hipoglicemia são obstáculos potenciais. A glicemia próxima do normal com risco mínimo de hipoglicemia, melhorar a qualidade de vida e retardar ou evitar as complicações micro e macrovasculares tardias estão entre os objetivos da assistência global aos indivíduos com DMl. Entretanto, a rotina desses pacientes é complicada pela necessidade variável de insulina, pela irregularidade na ingestão dos carboidratos, na realização de exercícios físicos ou em razão da presença de doenças intercorrentes.

As crianças e os adolescentes são um grupo especial, passam por diversos estágios de desenvolvimento físico e psicológico que demandam abordagem terapêutica dinâmica e atenta. Na puberdade, o padrão errático da alimentação e da atividade física é amplificado nos indivíduos diabéticos tipo l por rápidas mudanças nas doses de insulina associadas ao estirão de crescimento e à resistência a ação da insulina (RI). Apesar de os avanços e de as várias formulações de insulina disponíveis no momento, na maioria das vezes não se consegue atingir as metas de um ótimo/bom controle nesses jovens, nem para prevenir excursões na glicemia pós-prandial nem nas flutuações nos níveis de glicemia no DMl.

A RI é uma anormalidade metabólica comum no DM2, mas também está presente em indivíduos com DMl (2). Apesar da RI no DM2 estar geralmente associada à obesidade, à hipertensão, à dislipidemia e a outras desordens metabólicas, estudos têm demonstrado que adultos portadores de DMl com sobrepeso, assim como aqueles com peso normal, apresentam RI periférica e hepática. Clampe insulínico realizado em portadores de DMl não-obesos, com condições glicêmicas variadas, demonstram aumento da produção hepática de glicose e clearence de insulina reduzido comparados a indivíduos não-diabéticos (3). Por outro lado, pacientes portadores de DMl em tratamento insulínico intensivo que apresentam história familiar de DM2, também expressam marcadores da RI, tais como ganho de peso, necessidades maiores de insulina e dislipidemia (4).

Em virtude da insuficiência em se atingir os objetivos de um bom controle com as preparações de insulina disponíveis, a mudança do perfil de uma porcentagem dos portadores de DMl com a associação de alguns fatores de RI (sedentarismo e obesidade) estimulou o interesse em estudar agentes farmacológicos que pudessem colaborar com a terapia insulínica nesse grupo especial. $\mathrm{O}$ arsenal de drogas disponíveis para se associar a terapia com a insulina no DMl pode, didaticamente, ser dividido em três grupos de acordo com seu mecanismo de ação: 1. Sensibilizadores à ação da insulina (metformina e tiazolidinedionas); 2. Drogas que alteram a manipulação dos nutrientes no trato gastrintestinal (acarbose e amilina); 3. Outros agentes, como pirenzepina (que reduz a secreção de hormônio de crescimento (GH), IGF-l (que reduz a secreção de GH e tem ação hipoglicemiante) e análogos do GLP-1 (que estimulam a secreção de insulina de modo glicosedependente).

\section{SENSIBILIZADORES DA INSULINA}

\section{Metformina}

A RI nos adolescentes é bem documentada tanto em pacientes diabéticos (2) quanto em indivíduos não-diabéticos (5). Nos pacientes diabéticos, é responsável pela deterioração do controle glicêmico encontrado durante a puberdade. No DCCT, os adolescentes obtiveram hemoglobina glicada ( $\mathrm{HbAlc}$ ) $1 \%$ maior que nos adultos apesar de utilizarem mais insulina e apresentarem maior ganho de peso. A tríade maior HbAlc, maior dose de insulina e maior ganho de peso sugere que a insulina administrada era menos efetiva para manter o controle glicêmico (isto é, RI) neste grupo. Além disso, a secreção GH está aumentada neste período nos portadores de DMl. A insulina aplicada por via subcutânea ultrapassa a circulação portal, o que diminui o seu efeito intra-hepático na síntese da proteína GHBP, com conseqüente diminuição da ação do GH e dos níveis de IGFl. Com isso, não ocorre o feedback inibitório, havendo exagerada secreção de $\mathrm{GH}$, o que contribui para RI.

A insulina, mesmo dada em altas doses para pacientes portadores de DMl que apresentam RI, é ineficaz para o controle glicêmico e contribui para o ganho de peso dos pacientes. Além disso, o hiperinsulinismo decorrente da aplicação de grande quantidade de insulina exógena é um fator de risco para aterosclerose e mortalidade cardiovascular (6). Deste modo, diminuir a RI com reflexo na redução das doses de insulina, auxiliando para o bom controle, seria importante para o manejo do $\mathrm{DMl}$, principalmente na adolescência. 
A metformina é uma biguanida que tem sido usada no manejo do DM2 há mais de 40 anos. Melhora o controle glicêmico, aumentando principalmente a sensibilidade hepática (por meio da supressão da glicogenólise e inibição da gluconeogenese no fígado) e em menor extensão melhora a sensibilidade do músculo à insulina (maior captação periférica de glicose e armazenamento no músculo). Também tem ação diminuindo a oxidação dos ácidos gordurosos e a absorção de glicose intestinal, mas a contribuição desses efeitos na ação anti-hiperglicemiante é considerada pequena. Além disso, colabora na redução do colesterol, dos triglicérides e do peso corporal (7).

Os primeiros estudos avaliando o uso da metformina em indivíduos portadores de DMl foram realizados em crianças na década de 1960 (8). Em um estudo de curta duração (quatro semanas), a conclusão foi de que a metformina não adicionava nenhum efeito benéfico à insulina. Nos 30 anos subseqüentes surgiram muitos estudos com metformina, mas limitados a pacientes portadores de DM2. No final da década de 1990, surgem pesquisas, desta vez em adultos portadores de $\mathrm{DMl}$, demonstrando que a associação de metformina reduz em média $25 \%$ as doses diárias de insulina. Posteriormente, avaliando-se adultos com $\mathrm{DMl}$ e sobrepeso, um estudo randomizado, duplo-cego e placebo controlado demonstrou que a associação da metformina à terapia insulínica melhorou o controle glicêmico sem aumentar a freqüência de hipoglicemia (redução de $0,8 \%$ na $\mathrm{HbAlc}$ ) e diminuiu $17 \%$ a dose total de insulina, o que favoreceu a manutenção do peso dos pacientes (9). Na tentativa de se observar se o efeito de redução da dose total de insulina seria relativo à insulina basal ou prandial, um grupo europeu avaliou a associação da metformina à terapia com bomba de infusão de insulina subcutânea e constatou diminuição na dose da insulina referente ao componente basal, diminuição das glicemias pós-prandiais sem elevar a incidência de hipoglicemia comparando-se ao grupo-controle (10). Também demonstrou-se que a maior sensibilidade à insulina (SI), obtida por indivíduos portadores de DMI, que utilizaram a metformina por período prolongado de até dois anos, foi responsável pela maior satisfação e melhor qualidade de vida referida por esses pacientes (11).

O acúmulo de dados mostrando a segurança da metformina renovou o interesse de seu uso em adolescentes diabéticos ou não. Em adolescentes obesos, mesmo sem hiperglicemia, a metformina promoveu melhora da composição corporal [diminuição do índice de massa corporal (IMC), da circunferência da cintura e do tecido adiposo abdominal] e diminuição da insulinemia de jejum (12).

Estudos recentes mais sistemáticos, bem delineados, procuram selecionar adolescentes com DMl com sinais de RI (aumento de IMC, altas doses de insulina, controle metabólico insatisfatório) para adicioná-los à terapia com insulina. Em um estudo sueco, duplo-cego, placebo-controlado, constatou-se que a adição de dois gramas de metformina à terapia insulínica de adolescentes com DMl com mau controle metabólico (HbAlc, 9,5\%) resultou melhora da captação periférica de glicose. Os benefícios atingidos, avaliados por meio do clampe euglicêmico hiperinsulinêmico, foram tanto melhores quanto maior o grau de RI desses adolescentes (13). Outros autores avaliaram o papel da metformina sobre a SI por intermédio do teste de tolerância à glicose endovenosa (FSIGT), em adolescentes DMl com RI. Não houve diferença na SI medida pelo FSIGT, mas o grupo da metformina mostrou tendência de melhora da SI quando se ajustou para os níveis glicêmicos. Além disso, os indicadores clínicos, tais como diminuição das doses de insulina, diminuição do IMC, menor $\mathrm{HbAl}$ c comparados aos controles sinalizam melhora da SI. Não se sabe, no entanto, se essa melhora foi decorrente da diminuição da produção hepática de glicose promovida pela droga ou conseqüente à perda de peso que esses pacientes apresentaram. (14). Nosso grupo também estudou a adição de metformina ( 1 a 1,5 gra$\mathrm{ma} / \mathrm{dia})$, durante um ano, à terapia de múltiplas doses de insulina em 22 adolescentes portadores de DMl com sobrepeso, controle metabólico insatisfatório (HbAlc > 8\%) e pelo menos um sinal de RI (acanthosis nigricans, hipertensão arterial, síndrome dos ovários policísticos ou microalbuminúria). Os resultados demonstraram melhora da SI (aferida pela fórmula do eGDR - estimated glucose disposal rate) (15) com repercussão favorável no controle glicêmico (diminuição da $\mathrm{HbAlc}$ em 1,7\%) e nos fatores de risco cardiovascular (diminuição da pressão diastólica, diminuição da microalbuminúria e aumento discreto do HDL-colesterol) (16).

No entanto, o uso da metformina nos indivíduos com DMl é ainda muito controverso. Muitos autores questionam se nesses pacientes, sabidamente propensos a apresentar cetose, a referida vantagem de diminuir as doses de insulina justificaria o risco da acidose láctica. Acreditam que os dados disponíveis até o momento li- 
mitam a terapia conjunta da metformina com a insulina, no portador de DMl obeso, com mau controle glicêmico $(\mathrm{HbAlc}>8 \%)$ e que apresente sinais claros da síndrome metabólica (17). Por outro lado, aqueles que defendem a metformina, chamam a atenção para a ausência de acidose láctica em uma metanálise recente com mais de 35 mil pacientes/ano avaliados, alguns com patologia cardiovascular ou insuficiência renal. Concluem que a metformina é uma droga segura e que seria suficiente orientar os pacientes a suspendê-la antes da realização de exames contrastados ou durante infecções significativas $(18,19)$.

\section{Tiazolidinedionas}

As novas tiazolidinedionas (TZDs), como a pioglitasona e a rosiglitasona, são agentes anti-hiperglicemiantes utilizados de forma isolada ou conjugada a outras drogas orais ou à insulina no tratamento do DM2. Atuam como agonista do receptor PPAR $\gamma$, reduzindo a resistência à insulina. Sua ação é predominantemente periférica, nos tecidos adiposo e muscular, promovendo a diferenciação dos adipócitos em um adipócito menor e mais sensível à insulina (20). Estudos em modelos animais de DMl (camundongos NOD) demonstraram que as TZDs também apresentam ações antiinflamatórias e antiapoptóticas sobre as ilhotas pancreáticas (21).

Em seres humanos, a associação de rosiglitasona à terapia insulínica de pacientes com diabetes auto-imune latente do adulto (LADA) evidenciou melhora do peptídeo $\mathrm{C}$ de jejum após sobrecarga oral de glicose, sugerindo papel protetor das TZDs sobre a função das células-beta (22).

Em adultos com DMl com sobrepeso, a rosiglitasona associada à insulina melhorou o controle glicêmico e a pressão arterial sem a necessidade de aumento das doses de insulina, e no grupo-placebo a melhora do controle glicêmico ocorreu com aumento paralelo da insulina em $11 \%$. Os benefícios foram proporcionalmente melhores nos pacientes que apresentavam maior grau de RI. Vale ressaltar que apesar de não ocorrer diferenças entre os dois grupos quanto à prevalência de hipoglicemia ou ganho de peso, o grupo da rosiglitasona apresentou significativamente mais edema e anemia, sendo inclusive relatado um episódio de insuficiência cardíaca (23).

Em adolescentes portadores de DMl com sinais de RI e peso adequado, a associação de $30 \mathrm{mg}$ de pioglitasona à terapia insulínica não resultou melhora do controle glicêmico. Em um elegante estudo randomizado, placebo-controlado, ambos os grupos obtiveram discreta melhora da hemoglobina glicada sem diferença na dose da insulina, na freqüência de hipoglicemia, na presença de edema ou anemia. Nesse estudo, o grupo da pioglitasona apresentou o dobro de ganho de peso que o grupo placebo, sendo a retenção de fluidos a provável responsável por esse efeito indesejado (24).

É importante salientar que uma recente metanálise da rosiglitasona com pacientes portadores de DM2 chamou a atenção para o aumento significativo do risco de infarto do miocárdio (odds ratio $=1,43$ (IC $=1,03$ a $1,98 ; \mathrm{p}=0,03)$ e de morte por causas cardiovasculares (odds ratio $=1,64 ; \mathrm{IC}=0,98$ a 2,$74 ; \mathrm{p}=0,06)(25)$. Entretanto, essa área ainda permanece em discussão. Como outro estudo recente comparou a rosiglitasona com a pioglitasona e concluiu que esta diminuiu em $22 \%$ o risco de pacientes internados por infarto do miocárdio (26). De modo que o uso das TZDs em adolescentes ou jovens com DMl carece de segurança maior para sua utilização.

\section{DROGAS QUE MODULAM A ABSORÇÃO DE NUTRIENTES NO TRATO GASTRINTESTINAL}

A concentração da glicose sanguínea é determinada pela taxa de glicose que entra na circulação (taxa de aparecimento da glicose) e pela taxa de glicose que é removida da circulação (taxa de desaparecimento da glicose). A taxa de aparecimento de glicose é controlada pelo glucagon (sua supressão reduz a produção hepática de glicose), pela saciedade (redução da ingestão de nutrientes, reduz a glicose exógena) e pelo atraso no esvaziamento gástrico (atrasando a absorção de glicose exógena). Por sua vez, a taxa de desaparecimento da glicose está sob o controle da insulina e da captação de glicose pelos tecidos periféricos. No passado, somente a taxa de desaparecimento desta equação (a insulina) estava disponível para alterar o controle glicêmico. Atualmente, drogas que atuam na modulação da absorção dos nutrientes no intestino, como acarbose e amilina, podem contribuir para o equilíbrio da glicemia ao diminuir a taxa de aparecimento da glicose.

\section{Acarbose}

Acredita-se que a instabilidade glicêmica pós-prandial colabore para a patogênese das complicações, em especial as macrovasculares. Assim, a redução das excursões 
glicêmicas pós-prandiais pode ser um importante passo para diminuir o risco macrovascular. $\mathrm{O}$ manejo da hiperglicemia pós-prandial inclui a utilização de dieta rica em carboidrato de absorção lenta ou uso de drogas que atuem atrasando a absorção da sacarose. As alterações na alimentação, aumentando o conteúdo de fibras, são pouco efetivas em conseqüência de hábitos culturais e de baixa palatabilidade da dieta. A acarbose é uma droga que atrasa a hidrólise de dissacarídeos, olicassarídeos para monossacarídeos por meio da inibição competitivamente da enzima alfa-glucosidase existente na membrana intestinal. Em conseqüência dessa inibição, ocorre atraso da absorção de sacarose pela mucosa intestinal, o que contribui para reduzir a hiperglicemia pós-prandial. Além disso, o uso da acarbose é associada ao aumento do GLP-1 (glucagon-like peptide 1), o que pode contribuir para o efeito terapêutico dessa droga (27).

Em um estudo multicêntrico com adultos portadores de $\mathrm{DMl}$, a adição titulada de $100 \mathrm{mg}$ de acarbose, nas três principais refeições, produziu redução de 0,4 pontos percentuais na HbAlc. No entanto, 20\% dos pacientes abandonaram o estudo em razão de queixas gastrintestinais, tais como flatulência, diarréia e dor abdominal (28). Em outro estudo randomizado, placebocontrolado, com adição da acarbose, os indivíduos com DMl foram divididos de acordo com dieta rica ou pobre em fibras. Apesar de não ter havido nenhuma diferença na HbAlc entre os dois grupos, o grupo da acarbose apresentou menor glicemia pós-prandial, independentemente do conteúdo de carboidrato ou de fibras da dieta utilizada. Essa redução da hiperglicemia pós-prandial poderia ser benéfica na prevenção das complicações tardias do diabetes. Mas novamente a freqüência de efeitos colaterais gastrintestinais, apesar de considerados leves, foi significativamente maior no grupo da acarbose que do placebo ( $75 \%$ versus $39 \%$; $<<$ $0,02)(29)$.

Também foi pesquisado o efeito da acarbose sobre o exercício pós-prandial em indivíduos com DMl. A ingestão de $100 \mathrm{mg}$ de acarbose durante a refeição, realizada 90 minutos antes de exercício aeróbico, resultou redução significativa do aumento da glicemia pós-prandial e redução da hipoglicemia induzida pelo exercício, provavelmente em conseqüência do atraso na absorção da glicose e da menor produção hepática da glicose (30). Outros agentes inibidores da alfa-glucosidase mais recentes, que são quase completamente absorvidos no intestino delgado, como miglitol e voglibose, foram estudados em indi- víduos com DM2. Como mostraram semelhança na eficácia e nos efeitos adversos, não parecem ter vantagens adicionais sobre a acarbose.

Deve-se salientar que o uso de acarbose associado à insulina não está aprovado em nosso país. Entre outras justificativas, está o fato de que nos episódios de hipoglicemia nessa combinação há a necessidade de glicose para correção desde que a acarbose iniba a absorção de sacarose pela via intestinal.

\section{Amilina}

A amilina é um peptídeo com 37 aminoácidos, co-secretado com a insulina pelas células betapancreáticas, de modo pulsátil em resposta ao estímulo dos nutrientes. Em indivíduos saudáveis, os níveis plasmáticos de amilina variam de 4 a 8 pmol/L (jejum) e aumentam para 15 a $25 \mathrm{pmol} / \mathrm{L}$ (após a alimentação), sendo substancialmente menores que os níveis de insulina (razão molar de $1 / 20$ ). Seus receptores são uma combinação de isoformas do receptor de calcitonina com diferentes proteínas RAMPs (receptor activity-modifying proteins) e estão presentes no sistema nervoso central ( $\mathrm{NNC}$ ), músculo, rins, fígado e nas células betapancreáticas.

Os efeitos glicorreguladores da amilina incluem inibição do esvaziamento gástrico, da liberação do glucagon de modo glicose-dependente, da secreção de ácido gástrico e efeitos sacietógenos no SNC (redução da ingestão alimentar e do peso).

O análogo da amilina, o pramlintide, é uma droga sintética injetável, de excreção renal, do qual $40 \%$ ligam-se à albumina, apresentam pico plasmático aos 20 minutos e têm duração de ação em torno de três horas. A dose terapêutica varia de 30 a $60 \mu \mathrm{g}$, que corresponde à secreção normal pós-prandial da amilina, devendo ser administrada no subcutâneo separada da insulina, visto que apresenta $\mathrm{pH}$ de 4 (31). É aconselhado iniciar com $15 \mu \mathrm{g}$ antes das principais refeições (equivale a 2,5 $U$ na seringa de insulina) e aumentar $15 \mu$ g por semana até $60 \mu \mathrm{g}$ para reduzir náuseas e vômitos. Para minimizar os riscos de hipoglicemia, a insulina prandial deve ser diminuída em $50 \%$. Também é importante que a aplicação da insulina seja feita no meio ou no final da refeição, já que o pramlintide induz saciedade e torna o apetite e a ingestão de nutrientes pouco previsíveis $(32)$.

No paciente com DMl, a insulina e a amilina estão deficientes. Em um grande estudo multicêntrico, duplo-cego, placebo-controlado, com 651 pacientes por- 
tadores de $\mathrm{DMl}$, o uso de $60 \mu \mathrm{g}$ de pramlintide nas três refeições resultou uma modesta, mas significativa, redução da HbAlc em 0,3\%, melhora do controle glicêmico pós-prandial e diminuição do peso, especialmente nos pacientes obesos. O estudo chama a atenção para o aumento da freqüência de hipoglicemia grave nas primeiras semanas, no entanto, $o$ pramlintide não foi iniciado com baixas doses nem a insulina prandial foi diminuída. O grupo de tratamento apresentou freqüência duas vezes maior de náuseas, anorexia e vômitos de leve a moderada intensidade nas primeiras semanas (33). A melhora da glicemia pós-prandial ocasionada pelo pramlintide resulta não somente do atraso do esvaziamento gástrico, mas principalmente da diminuição da secreção do glucagon pós-alimentar (34).

Muitos adolescentes com DMl apresentam instabilidade glicêmica com hiperglicemia pós-prandial de difícil controle. A tentativa de correção por meio do ajuste da dose de insulina seja na terapia com múltiplas doses de insulina seja com a bomba de infusão pode resultar hipoglicemia tardia. A associação do pramlintide nessa terapêutica reduz significativamente esse problema. Ao reduzir a secreção de glucagon, o pramlintide provoca diminuição imediata da glicemia (nadir ocorrendo após 45 a 60 minutos) e possibilita o uso de doses menores da insulina prandial, o que evita a hipoglicemia $(35,36)$. Está demonstrado que esse efeito não é mediado pelo glucagon-like peptide 1 (GLP-1) (37).

\section{OUTROS AGENTES}

\section{Pirenzepina}

O aumento da secreção do hormônio de crescimento $(\mathrm{GH})$ pode ser um dos fatores responsáveis pela instabilidade glicêmica comum na adolescência, especialmente nas meninas. $\mathrm{O}$ GH interfere tanto na sensibilidade insulínica hepática quanto na periférica. Comparado aos adolescentes saudáveis, o GH está elevado e a IGF-1 diminuída nos diabéticos. O GH é secretado de modo pulsátil pela hipófise sob regulação hipotalâmica, sendo estimulado pelo GHRH e inibido pela somatostatina. Esta, por sua vez, está sob controle do sistema anticolinérgico por meio dos receptores muscarínicos.

Desde a década de 1990, muitos estudos utilizando anticolinérgicos para reduzir o GH e melhorar o controle glicêmico foram realizados. A avaliação do trata- mento insulínico associado à $100 \mathrm{mg}$ de pirenzepina (PZP) em meninas com DMl, por intermédio do clampe euglicêmico hiperinsulinêmico, demonstrou melhora da sensibilidade insulínica, mas surpreendentemente não à custa da redução da secreção de $\mathrm{GH}$. $\mathrm{O}$ autor interroga se a melhora da resposta glicêmica não seria decorrente da diminuição do polipeptídeo pancreático (PP), que é regulado pelo sistema colinérgico. A diminuição do PP reduz a motilidade gastrintestinal, o que diminui a oferta de glicose (38). Isto contrasta com outros estudos nos quais diminuição significativa do GH foi observada (39).

\section{IGF-1}

A insulina é considerada o principal hormônio hipoglicemiante, mas sabe-se que o potencial do sistema IGF para diminuir os valores de glicemia é 50 vezes maior que a insulina. Entretanto, esse potencial hipoglicemiante é prejudicado pelo fato de que apenas uma pequena fração da IGF circula livremente, estando o restante ligado às proteínas: as IGFBPs.

Os baixos níveis de insulina no sistema-porta dos diabéticos elevam a IGFBP-1, que é uma proteína que inibe da ação da IGF-1, de modo que os baixos níveis circulantes da IGF- 1 contribuem para a piora do controle metabólico. Somente a administração direta da insulina no sistema-porta pode restaurar essas anormalidades. Entretanto, observações que a correção dos níveis de IGF-1 com IGF-1 humano recombinante (rhIGF-1) diminui o GH, melhora a sensibilidade à insulina e reduz a necessidade insulínica para a euglicemia, estimularam o interesse no potencial do rhIGF-l como terapia adjuvante à insulina nos indivíduos DMl (40).

A administração subcutânea de rhIGF-1 associado à terapia insulínica convencional em uma população de 223 indivíduos com DMl demonstrou queda significativa da hemoglobina glicada com menores doses de insulina, comparando-se ao grupo que intensificou o tratamento insulínico. No entanto, observou-se que os grupos randomizados com as maiores doses de rhIGF-1 ( $80 \mu \mathrm{g} / \mathrm{kg}$, duas vezes ao dia) apresentaram piora significativa da retinopatia e outros efeitos adversos menores também dose-dependentes, como edema periférico, dor na mandíbula e taquicardia (41). Apesar de a dose de $40 \mu \mathrm{g} / \mathrm{kg}$ mostrar-se segura e adequada para otimizar o tratamento insulínico, mais estudos são necessários para determinar o risco-benefício dessa nova terapia adjunta. 


\section{Análogos do GLP-1}

A alimentação provoca a secreção de múltiplos hormônios gastrintestinais envolvidos na regulação da motilidade intestinal, na secreção de ácido gástrico e das enzimas pancreáticas, na contração da bexiga e na absorção de nutrientes. A observação que a nutrição enteral seja um estímulo insulinotrópico mais potente comparado com o estímulo intravenoso isoglicêmico levou ao conceito de incretinas.

A primeira incretina identificada foi a glucose-dependent insulinotropic polypeptide (GIP), um peptídeo de 42 aminoácidos secretado pelas células $\mathrm{K}$ localizadas principalmente no duodeno. Foi purificada de extrato intestinal de porco, mostrando fracos efeitos sobre a secreção ácida do estômago, mas com efeitos insulinotrópicos mais potentes em seres humanos. $\mathrm{O}$ segundo hormônio incretínico identificado foi o glucagon-like peptide 1 (GLP-1), produzido pelas células L do íleo distal e cólon sendo liberado após a ingestão alimentar. Hoje considerado a principal incretina, o GLP-1 age nas ilhotas pancreáticas tanto nas células-beta (estimulando a síntese de insulina) quanto nas células-alfa (inibindo a secreção de glucagon). Essa ação é dependente da glicemia, o que evita a hipoglicemia. Além disso, inibe o esvaziamento gástrico por meio de ação local e hipotalâmica e diminuiu o apetite (ação hipotalâmica) (42).

Estudos promissores em modelos animais revelam que o GLP-1 também é capaz de promover expansão da massa de células-beta, inibição da apoptose dessas células e estimular a diferenciação de células germinativas do epitélio ductal por meio da neogênese das ilhotas (43). Nos seres humanos, os estudos demonstram que essas ações proliferativas e antiapoptóticas podem contribuir para um papel protetor ou mesmo regenerativo do GLP-1 sobre as células-beta. Deste modo, pesquisadores canadenses avaliaram o efeito do GLP-1 sobre a secreção de insulina de pacientes com DMl que receberam transplantes de ilhotas e demonstraram que as células-beta eram capazes de responder ao GLP-1. Os autores sugerem que esse peptídeo pode desempenhar importante papel no controle glicêmico e na manutenção da massa de célula-beta de pacientes transplantados (44). O papel dos outros peptídeos produzidos, como o GLP-2, a oxintomodulina, ainda não está claro na prática clínica endocrinológica.

Pouco se conhece até o momento sobre as incretinas na história natural do DMl. Alguns estudos foram realizados em indivíduos com risco para DMl, em pacientes recém-diagnosticados e em pacientes com doen- ça clínica estabelecida (45). No primeiro grupo, foram estudados parentes de primeiro e segundo graus de pacientes portadores de $\mathrm{DMl}$, que apresentavam anticorpos antiilhotas positivos e demonstravam hiperglicemia somente após o teste de tolerância oral à glicose (isto é, a glicemia de jejum era normal) no Diabetes Prevention Trial (DPT-1). A avaliação desses indivíduos permitiu determinar quais eram os fatores que permitiam a manutenção da euglicemia de jejum enquanto incapazes de regular a glicemia pós-sobrecarga oral de glicose. O que chamou a atenção foi o fato de, apesar de os hormônios GLP-1 e GIP estarem normais, a resposta insulínica e a ingestão de glicose (o efeito incretínico) foi subnormal e não ocorreu a adequada supressão da secreção do glucagon durante a hiperglicemia. Demonstrou-se, assim, que o prejuízo do efeito incretínico é uma alteração precoce no DMl (46).

Os mecanismos enteroinsulares também mostraram-se importantes no controle glicêmico do DMl recém-diagnosticado. Estudando voluntários com DMl com reserva endógena de insulina (na qual é possível a descontinuação da insulina sem prejuízo do controle glicêmico), o uso de glucagon parenteral demonstrou que apesar de a insulina não responder à glicose parenteral, a resposta à refeição mista é preservada (47).

A ação hipoglicemiante do GLP-l em indivíduos portadores de DMl é elegantemente demonstrada em um estudo por intermédio da infusão endovenosa desse peptídeo durante hiperglicemia, no período pós-absortivo e no jejum. Sem a administração concomitante de insulina, a infusão do GLP-1 diminuiu a glicemia para níveis normais, e isto foi associado à inibição da secreção de glucagon (48). Quando a infusão prolongada de GLP-1 foi combinada à insulina (para manter as variações glicêmicas dentro de uma faixa fisiológica), tanto as incursões glicêmicas como as doses de insulina reveladas foram reduzidas quando comparadas às do grupo que recebeu insulina isoladamente. Esses achados demonstram que o GLP-1 também melhora a sensibilidade à insulina (49).

Apesar de muito deste efeito poder ser justificado pelo aumento no tempo de esvaziamento gástrico, a infusão de GLP-1 suprime de modo dose-dependente a secreção anormal do glucagon e do polipeptídeo pancreático (PP) que ocorre após a ingestão alimentar nos indivíduos com DMl, e isto contribuiu para melhorar a hiperglicemia pós-prandial, especialmente dos pacientes com peptídeo C negativo (50-52). 
De modo que, muitos estudos estão sendo desenvolvidos com as drogas incretinomiméticas em pacientes com DMl, inclusive crianças, para a confirmação dos reais benefícios desses novos agentes (53).

\section{CONCLUSÕES}

As dificuldades encontradas para se atingir a euglicemia em pacientes portadores de DMl, especialmente durante a resistência à insulina que ocorre na adolescência e nos pacientes que apresentam sinais da síndrome metabólica, têm incentivado pesquisas com novas drogas que minimizem a hiperglicemia pós-prandial. Além disso, a terapia intensiva com insulina é associada a um risco de ganho de peso excessivo. De todas as drogas estudas, somente a metformina foi suficientemente testada quanto à segurança $\mathrm{e}$ à eficácia. No entanto, outros agentes promissores estão em estudo com o objetivo de aumentar a saciedade e diminuir a secreção pós-prandial de glucagon reduzindo, assim, a hiperglicemia pós-prandial. Assim, estudo de longo prazo com o análago do GLP-1 ou com o GLP-1 em bomba de infusão subcutânea contínua associado à terapia insulínica e devem ser implementados para confirmar a melhora do controle glicêmico sem o risco inaceitável de hipoglicemia e ganho de peso. Além disso, estudos em indivíduos com DMl ainda em fase de remissão e pacientes submetidos aos transplantes de pâncreas total ou de ilhotas devem esclarecer o potencial trófico do GLP-1 para as células-beta.

\section{REFERÊNCIAS}

1. The Diabetes Control and Complications Trial Research Group: The effect of intensive treatment of diabetes on the development and progression of long term complications in insulindependent diabetes mellitus. N Engl J Med. 1993;329:977-86.

2. Dib SA. Resistência à insulina e síndrome metabólica no diabetes melito do tipo 1. Arq Bras Endocrinol Metab. 2006;50(2): 250-63.

3. Makimattila S, Virkamaki A, Malmstrom R, Utriainen T, YkiJarvinen H. Insulin resistance in type 1 diabetes mellitus: a major role for reduced glucose extraction. J Clin Endocrinol Metab. 1996;81:1036-40.

4. Pumell JQ, Dev RK, Steffes MW, Cleary PA, Palmer JP, Hirsch IB, et al. Relation of family history of type 2 diabetes, hypoglycemia and autoantibodies to weight gain and lipids with intensive and conventional therapy in the Diabetes Control and Complications Trial. Diabetes. 2003;52:2623-96.

5. Rocco ER, Mory DB, Silva RCQ, Gabbay MAL, Komatsu WR, Dib SA. Síndrome Metabólica através do espectro da resistência à insulina em adolescentes com tolerância normal a glicose. Arq Bras Endocrinol Metab. 2007;51(3):S91.
6. Wiernsperger NF, Bailey CJ. The antihyperglycaemic effect of metformin: therapeutic and cellular mechanisms. Drugs. 1999;58(suppl 1):31-9.

7. Wei M, Gaskill SP, Haffner SM, Stern MP. Effects of diabetes and level of glycemia on all-cause and cardiovascular mortality: the San Antonio Heart Study. Diabetes Care. 1998;21:1167-72.

8. Ferguson AW, de La Harpe PL, Farquhar JW. Dimethydiguanide in the treatment of diabetic children. Lancet. 1961;1:1367-9.

9. Khan ASA, McLougney CR, Ahmed AB. The effects of metformin on blood glucose control in overweight patients with Type 1 diabetes. Diabet Med. 2006; 23:1079-84.

10. Meyer L, Bohme P, Delbachian I, Lehert P, Cugnardey N, Drouin $\mathrm{P}$, Guerci $\mathrm{B}$. The benefits of metformin therapy during continuous subcutaneous insulin infusion treatment of type 1 diabetic patients. Diabetes Care. 2002;25:2153-8.

11. Moon R.J., Bascombe LA and Holt R.I.G. The adittion of Metformin in Type 1 Diabetes improves insulin sensitivity, diabetic control, body composition and patient well- being. Diabet Obes Metab. 2007;9:143-5.

12. Srinivasan S, Ambler GR, Baur LA, Garnett SP, Tepsa M, Ward $\mathrm{GM}$, et al. Randomized, controlled trial of metformin for obesity and insulin resistance in children and adolescents: improvement in body composition and fasting insulin. J Clin Enocrinol Metab. 2006;91:2074-80.

13. Sarnblad S, Kroon M, Aman J. Metformin as an additional therapy in adolescents with poorly controlled type 1 diabetes: randomized placebo controlled trial with aspects on insulin sensitivity. Eur J Endocrinol. 2003;149:323 -9.

14. Hamilton J, Cummings E, Dravkovic V, Finegood D, Daneman D. Metformin as an adjunt therapy in adolescents with type 1 diabetes and insulin resistance. Diabetes Care. 2003;26:138-43.

15. Williams KV, Erbey JR, Becker D, Arslanian S, Orchard TJ. Can clinical factors estimate insulin resistance in type 1 diabetes? Diabetes. 2000;49:626-32.

16. Gabbay MAL, Dualibi P, Rocco E, Dib SA. Metformina melhora a sensibilidade à insulina e o controle glicêmico do diabetes melito tipo $1 \mathrm{com}$ sobrepeso. Arq Bras Endocrinol Metab. 2007;51(3)(supl 1):S86.

17. Faichney JD, Tate PW. Metformin in type 1 diabetes. Is this a good or bad idea? Diabetes Care. 2003;26:1655.

18. Masoudi FA, Wang Y, Inzucchi SF, Setaro JF, Havranek EP, Foody JM, Krumholz HM. Metformin and thiazolidinedione use in Medicare patients with heart failure. JAMA. 2003;290:81-5.

19. Aldasouqi SA, Duick DS. Safety issues on metformin use. Diabetes Care. 2003;26:3356.

20. Yki Jarvinen H. Thiazolidinediones. N Engl J Med. 2004;351: 1106-18.

21. Beales PE, Pozzili P. Thiazolidinediones for the prevention of diabetes in the non-obese diabetic (NOD) mouse: implications for human type 1 diabetes. Diabetes Metab Res Rev. 2002;18:114-7.

22. Zhou Z, Li X, Huang G, Peng J, Yang L, Yan X, et al. Rosiglitazone combined with insulin preserves islet beta function in adult-onset latent autoimmune diabetes (LADA). Diabetes Metab Res. 2005; 21:203-8.

23. Strowig S, Raskin P. The effects of rosiglitazone on overweight subjects with type 1 diabetes. Diabetes Care. 2005;28:1562-7.

24. Zdravkovic V, Hamilton J, Daneman D, Cummings E. Pioglitazone as adjunctive therapy in adolescents with type 1 diabetes. J Pediatr 2006; 149:845-9.

25. Nissen, Steven E, Wolski, Kathy. Effect of Rosiglitazone on the risk of myocardial infarction and death from cardiovascular causes. N Engl J Med. 2007;356:2457-71. 
26. Gerrits CM, Bhattacharya M, Manthena S, Baran R, Perez A, Kupfer S. A compararison of pioglitazone and rosiglitazone for hospitalization for acute myocardial infarction in type 2 diabetes. Pharmacoepidemiol Drug Saf. 2007;16:1065-71.

27. Goke B, Herrmann C, Goke R, Fehmann HC, Berghofer P, Richter $\mathrm{G}$, et al. Intestinal effects of alpha-glucosidase inhibitors: absorption of nutrients and enterohormonal changes. Eur J Clin Invest. 1994;24 (suppl 3):25-30.

28. Sels JPJE, Verdonk HER, Wolffenbuttel BHR. Effects of acarbose (Glucobay $\left.{ }^{\circledR}\right)$ in person with type 1 diabetes: a multicentre study. Diab Res Clin Prac. 1998;41:139-45.

29. Riccardi G, Giacco R, Parillo M, Turco S, Rivellese AA, Ventura $M R$, et al. Efficacy and safety of acarbose in the treatment of type 1 diabetes mellitus: a placebo-controlled, double-blind, multicentre study. Diabet Med. 1999;16:228-32.

30. Rabasa-Lhoret R, Burelle $Y$, Ducros F, Bourque J, Lavoie C, Massicotte $D$, et al. Use of an $\alpha$-glucosidase inhibitor to maintain glucose homoeostasis during post prandial exercise in intensively treated type 1 diabetes subjects. Diabet Med. 2001;18:739-744.

31. Weyer C, Maggs DG, Young AA. Amylin replacement with pramlintide as an adjunct to insulin therapy in type 1 and type 2 diabetes mellitus: a physiological approach toward improved metabolic control. Curr Pharm Des. 2001;7:1353-73.

32. Edelman SV, Caballero L. Amylin replacement therapy in patients with type 1 diabetes. Diabetes Educator. 2006;32:119s$127 \mathrm{~s}$.

33. Ratner RE, Dickey R, Fineman M, Maggs DG, Shen L, Strobel $\mathrm{SA}$, et al. Amylin replacement with pramlintide as an adjunct to insulin therapy improves long-term glycaemic and weight control in type 1 diabetes mellitus: a 1 year randomized controlled trial. Diabet Med. 2004;21:1204-12.

34. Fineman MS, koda JE, Shen LZ, Strobel SA, Maggs DG, Weyer $C$, et al. The Human Amylin Analog, Pramlintide, corrects postprandial Hyperglucagonemia in patients with type 1 diabetes. Metabolism. 2002; 51:636-41.

35. Heptulla RA, Rodriguez LM, Bomgaars L, Haymond MW. The role of Amylin and Glucagon in the Dampening of Glycemic excursions in children with Type 1 Diabetes. Diabetes. 2005;54:1100-07.

36. Edelman S, Garg S, FriasJ, Maggs D, Wang Y, Zhang B, et al. A double blind placebo-controlled trial assessing Pramlintide treatment in the setting of Intensive Insulin Therapy in Type 1 Diabetes. Diabetes Care. 2006;29:2189-95.

37. Ahrén B, Adner N, Svartberg, Petrella E, Holst JJ, Gutniak MK. Anti-diabetogenic effect of the human amylin analogue, pramlintide, in Type 1 diabetes is not mediated by GLP-1. Diabet Med. 2002;19:790-6.

38. Halldin UM, Brismar K, Tuvemo T, Gustafsson J. Insulin sensitivity and lipolysis in adolescent girls with poorly controlled type 1 diabetes: effect of anticholinergic treatment. Clin Endocrinol. 2002; 57:735-43.

39. Âman J, Kroon M, Karlsson L, Jones I, Hagenäs L. Reduced growth hormone secretion improves insulin sensitivity in adolescent girls with type 1 diabetes. Acta Paediatr. 1996; 85:31-71.
40. Carroll PV, Christ ER, Umpleby M, Gowrie I, Jackson N, Bowes SB, et al. IGF-1 Treatment in adults with type 1 diabetes - efftects on glucose and protein metabolism in the fasting state and during a hyperinsulinemic-euglycemic amino acid clamp. Diabetes. 2000; 49:789 -96.

41. Thrailkill KM, Quattrin T, Baker L, Kuntze JE, Compton PG, Martha Jr PM. Cotherapy with recombinant human insulin-like growth factor $\mathrm{i}$ and insulin improves glycemic control in type 1 diabetes. Diabetes Care. 1999;22:585-92.

42. Drucker DJ. The role of gut hormones in glucose homeostasis. J Clin Invest. 2007; 117:24-32.

43. Brubaker PL, Drucker DJ. Minireview: Glucagon-Like peptides regulate cell proliferation and apoptosis in the pancreas, gut, and central nervous system. Endocrinology. 2004;145:2653-9.

44. Fung M, Thompson D, Shapiro RJ, Warnock G, Andersen DA, Elahi D, et al. Effect of glucagon-like peptide -1 (7-37) on betacell function after islet transplantation in type 1 diabetes. Diab Res Clin Pract 2006; 74:189-93.

45. Dupre J. Glycaemic effects of incretins in type 1 diabetes mellitus. A concise review, with enphasis on studies in humans. Regul Pept. 2005;128:149-57.

46. Greenbaum CJ, Prigeon RL, D'Alessio DA. Impaired $\beta$-Cell function, incretin effect, and glucagon suppression in patients with type 1 diabetes who have normal fasting glucose. Diabetes. 2002; 51:951-7.

47. Mahon JL, Dupre J, Stiller, Donner AP. Immuno-supression in IDDM: rationale, risks, benefits and strategies. Diabetes Care. 1996;13:806-9.

48. Creutzfeldt WO, Kleine N, Willms B, Orskov C, Holst JJ, Nauck MA. Glucagonostatic actions and reduction of fasting hyperglycemia by exogenous glucagon-like peptide I (7-36) aide in type 1 diabetic patients. Diabetes Care. 1996;19:580-6.

49. Gutniak M, Orskov C, Holst JJ, Aren B, Efendic S. Antidiabetogenic effect of glucagon-like peptide -1 (7-36) amide in normal subjects and patients with diabetes mellitus. N Engl J Med. 1992;326:1316-22.

50. Dupre J, Behme MT, Hramiak IM, McFarlane P, Williamson MP, Zabel P, et al. Glucagon-like peptide I reduces postprandial glycemic excursions in IDDM. Diabetes. 1995;44:626-30.

51. Behme MT, Dupre J, McDonald TJ. Glucagon-like peptide 1 improved glycemic control in type 1 diabetes. BMC Endocr Disord. 2003;3:3.

52. Dupre J, Behme MT, McDonald TJ. Exendin-4 normalized post cibal glycemic excursions in type 1 diabetes. J Clin Endocrinol Metab. 2004;3469-73.

53. Jeha GS, Heptula RA. Newer therapeutic options for children with diabetes mellitus: theoretical and practical considerations. Pediat Diab. 2006;7:122-38.

Endereço para correspondência :

Mônica de Andrade Lima Gabbay

Rua Botucatu, 740

04023-900 São Paulo, SP

E-mail: gabbay@ajato.com.br 